\title{
Using the metabolism model of GH-Method: math- physical medicine to study geriatric concerns
}

\section{Introduction}

This paper addresses certain geriatric issues by using GHMethod: math-physical medicine (MPM) approach, particularly the mathematical metabolism model developed by the author in 2014 .

\section{Methods and results}

As shown in Figure 1, the chronic diseases distribution map of adults over 65 years is as follows from CDC (2007-2008):

\section{Endocrine chronic diseases:}

Hypertension 56\%

Heart diseases $33 \%$

Cancer 23\%

Diabetes $19 \%$

Stroke $9 \%$

Non-endocrine chronic diseases:

Arthritis 49\%

Asthma 11\%

\section{Bronchitis 9\%}

It is obvious that endocrine-related chronic diseases are the major concerns. The author has been living with severe type 2 diabetes for 25 years and has suffered five cardiac episodes, renal complications, retinal problems, bladder infections, foot ulcer, and thyroid issues. Therefore, he started to self-study diabetes since 2010. In 2014, he

\author{
Volume 5 Issue 3 - 2020
}

\section{Gerald C Hsu}

Medical Research Scientist, eclaireMD Foundation, USA

Correspondence: Gerald C Hsu, Medical Research Scientist, eclaireMD Foundation, USA, Email g.hsu@eclairemd.com

Received: May 05, 2020 | Published: May 13, 2020 utilized his learned medical knowledge of metabolic disorders with finite-element modeling technique of structural and mechanical engineering and advanced mathematics to develop a mathematical metabolism model. This model contains 10 categories (Figure 2: body output: weight, glucose, blood pressure, lipid; body input: food, exercise, sleep, stress, water drinking, routine life patterns) and $\sim 500$ detailed elements to calculate two dynamic variables, metabolism index (MI) and general health status unit (GHSU). Based on continuous data input, an APP on the smartphone can show both MI and GHSU continuously and dynamically on the screen to inform patients about their general health status, especially endocrine-related chronic disease conditions through body outputs (Figure 3). By using his research results and developed tool, his overall chronic disease conditions and complications, caused by his elevated glucose and $\mathrm{HbA1C}$, are all under control (Figure 4).

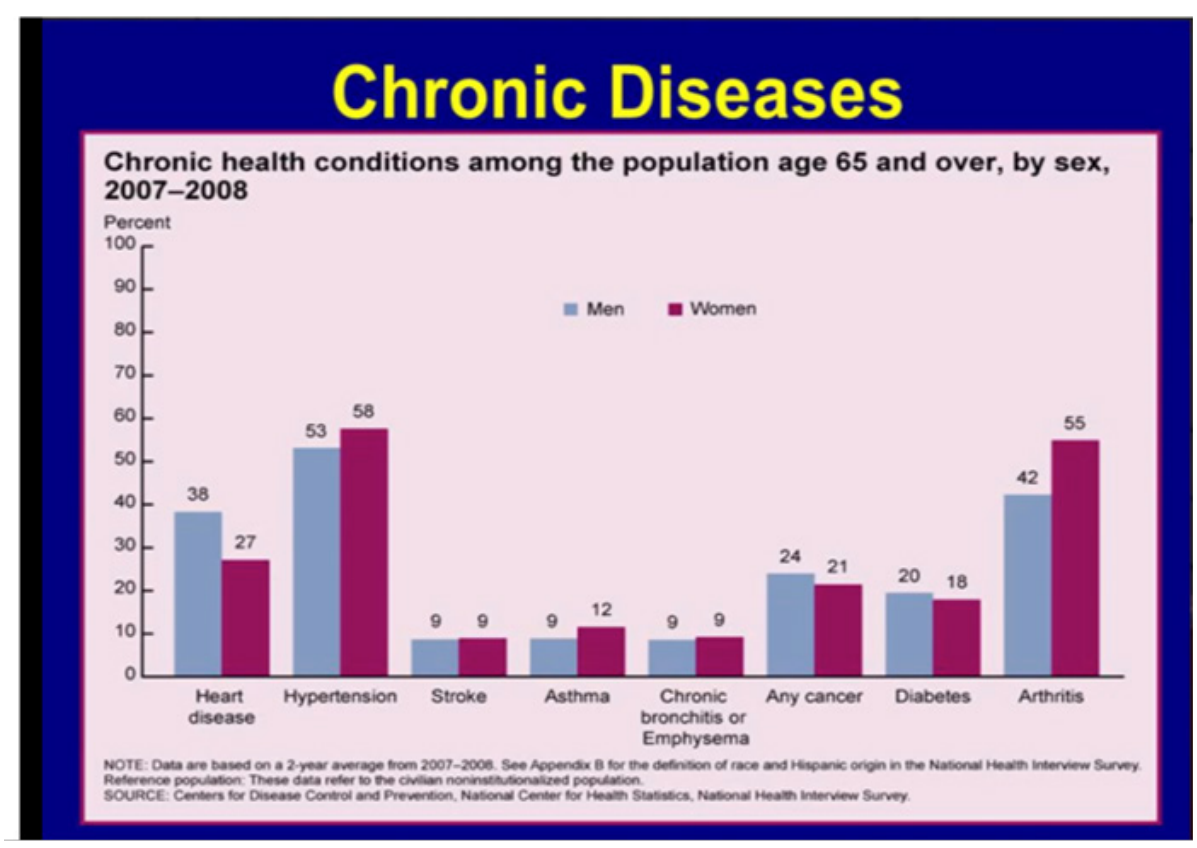

Figure I Chronic diseases among age >65 (2007-2008) . 


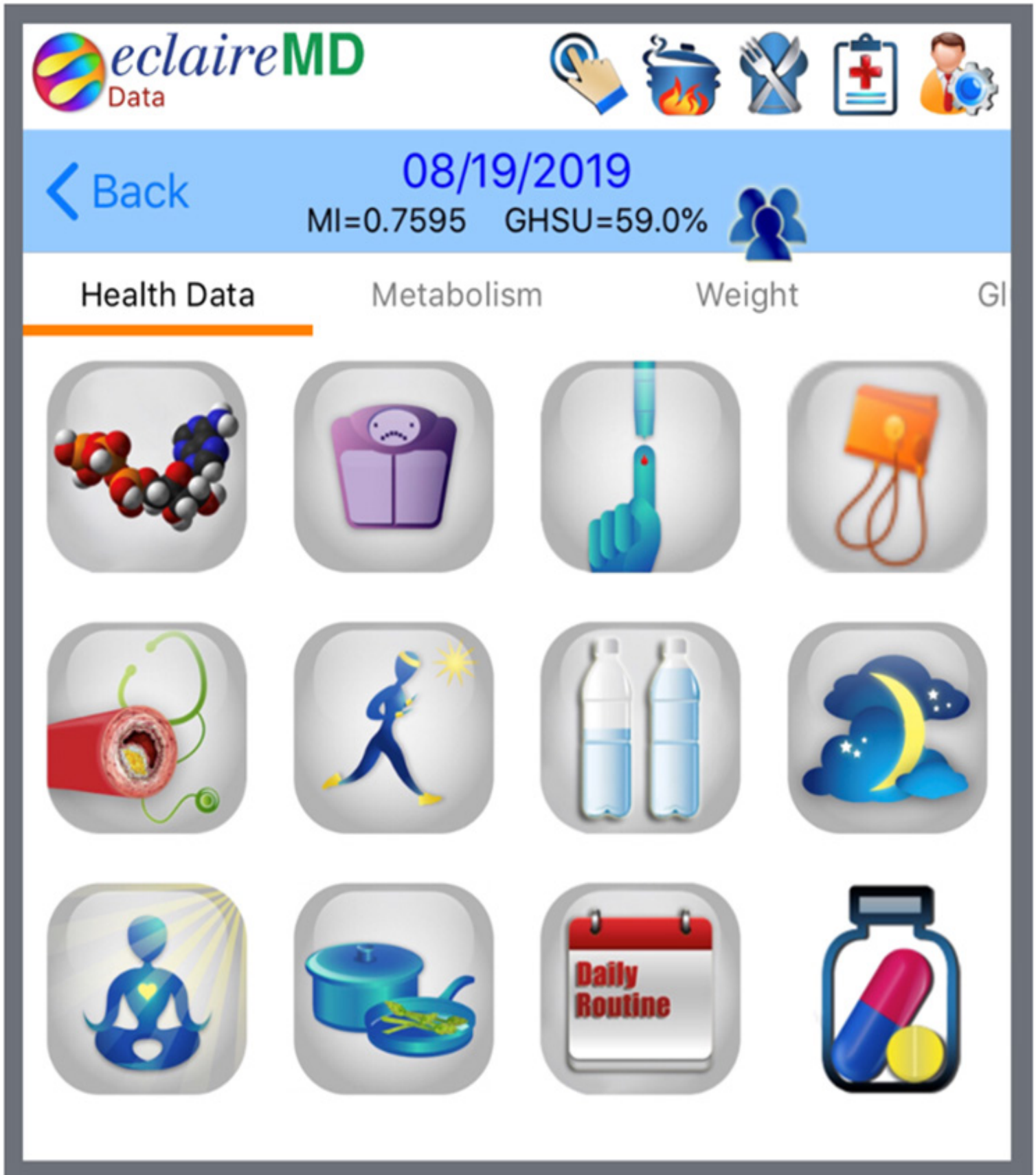

Figure 210 categories of metabolism model.

Since the author is over 72 years old, in 2019 , he started to selfstudy and research on geriatric diseases and disorders. He found that his developed metabolism model is extremely useful for older adults (geriatric population) as well as keeping their health conditions under control. The major differences between younger adults and older adults are in the areas of aging influences and "abnormal" metabolic conditions associated with aging. Therefore, the author has decided to expand and also augment his AI system within three selected categories (exercise, regular check-up, routine life pattern and home safety) to cover those geriatric concerns.
First, the pathophysiological changes are quite similar to his original chronic diseases design (items 1 through 9). He added in bones/muscle/dental/skin changes (10), five sensory changes (11), and mental/psychological changes (12) as shown below:

Routine check-up of geriatrics disorders:

Pathophysiological Changes:

(1) cardiovascular, cerebrovascular 

(2) endocrine (glucose, BP, lipids)
(3) brain, nervous system (dementia, pain)
(4) mental functions (depression)
(5) gastrointestinal
(6) pulmonary
(7) renal, urinary, reproductive
(8) sleep disorders

(9) nutritional status \& vitamin supplements

(10) osteoporosis, musculoskeletal, dental, integumentary

(11) sensory changes (vision, hearing, taste, smell, touch)

(12) emotional and psychological changes

(13) Special notes for geriatrics: medications review (should be $<$ four different kinds \& reduced dosage), BMI (should be $>20$, no lower).

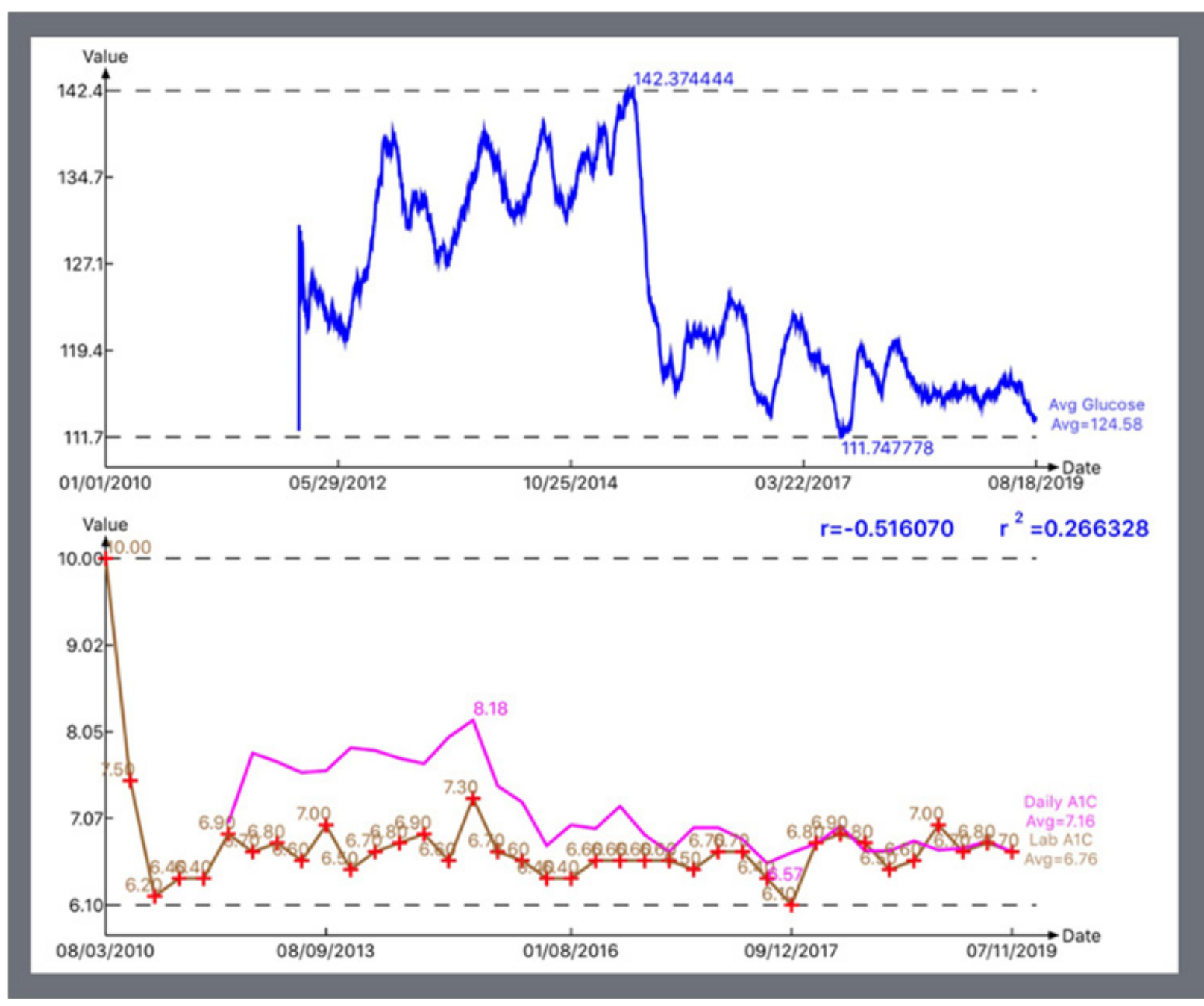

Figure 3 Metabolism Index (MI) \& General Health Status Unit (GHSU).

Second, in order to accommodate additional five people exercise requirements for geriatric concerns, he expanded his daily routine major exercise for walking to include tai-chi, yoga, water sports, and foot care in order to strengthen body balance, joint flexibility, and muscle strength (see list below).

Exercise for senior adults:
(14) maintain major daily exercise (e.g. walking)

(15) balance \& joint flexibility (e.g. tai chi, yoga, water sports)

(16) muscle and bone strength

(17) feet, toenails, comfortable shoes

(18) physical therapy if necessary. 


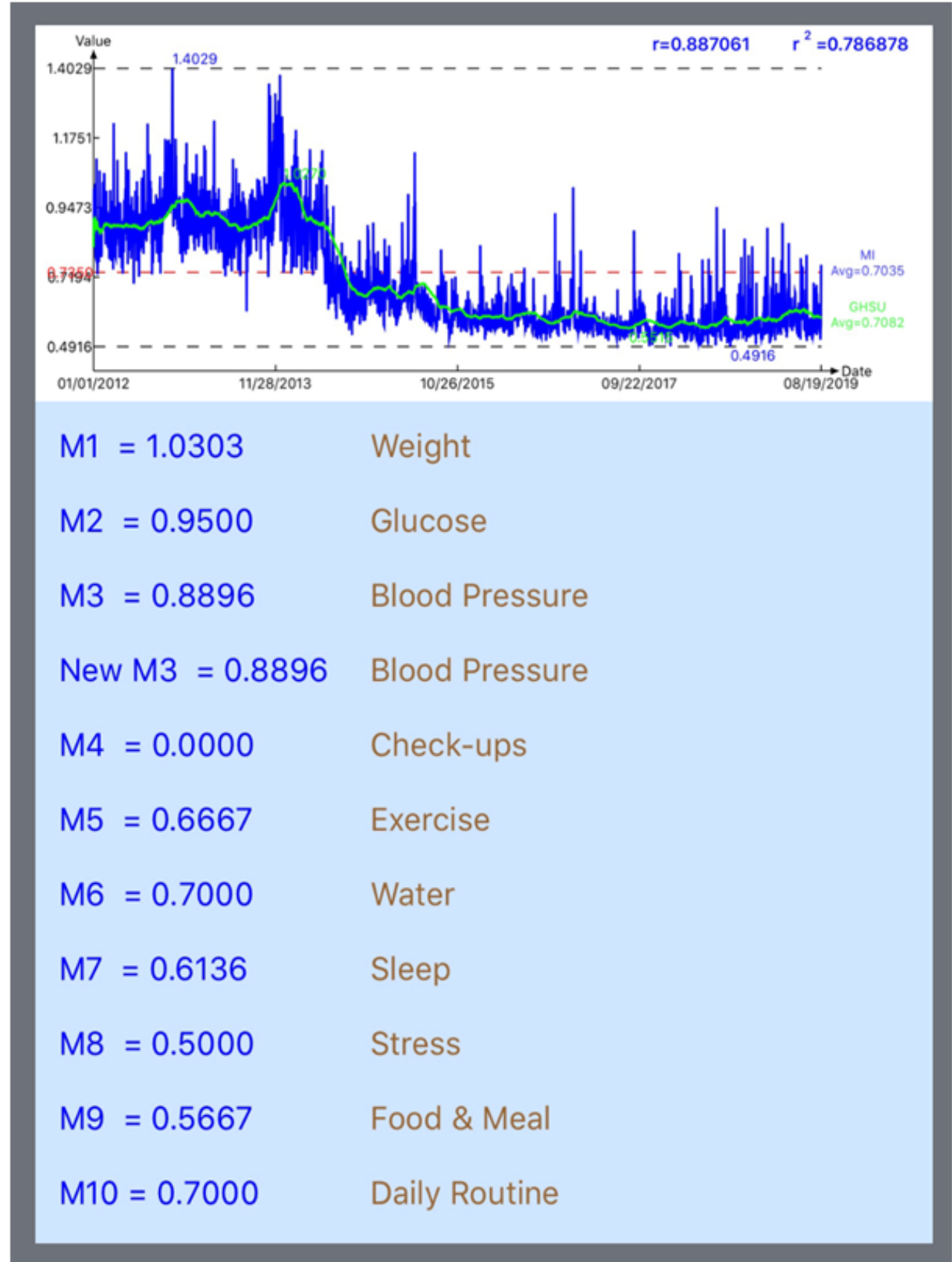

Figure 4 Daily glucose and HbAIC (2010-20I9).

Third, in order to prevent accidental injury or even fatality from falling, he added in the following eight "Home Safety" items into his "Routine Life Patterns".

Home safety for senior adults:

(19) avoid cords, wires, loose rugs

(20) install grab rails, fall proof devices

(21) safe bath/shower room, use shower chair \& bath brush for back and lower legs
(22) good lighting

(23) air conditioner and humidifier

(24) cane or walker, walk on smooth and non-slippery pavements

(25) caution for steps/stairs and moving traffic safety

(26) have an emergency alarm or alert system linked to caretaker or healthcare personnel

(27) have easy access for ambulance 
The geriatric items mentioned above are included to serve as either a daily status check or a reminder for seniors to follow or to install them. Through the APP on handheld devices, the overall quality of health and life extension of a senior citizen can be easier monitored and then maintained (Figure 5) (Figure 6).

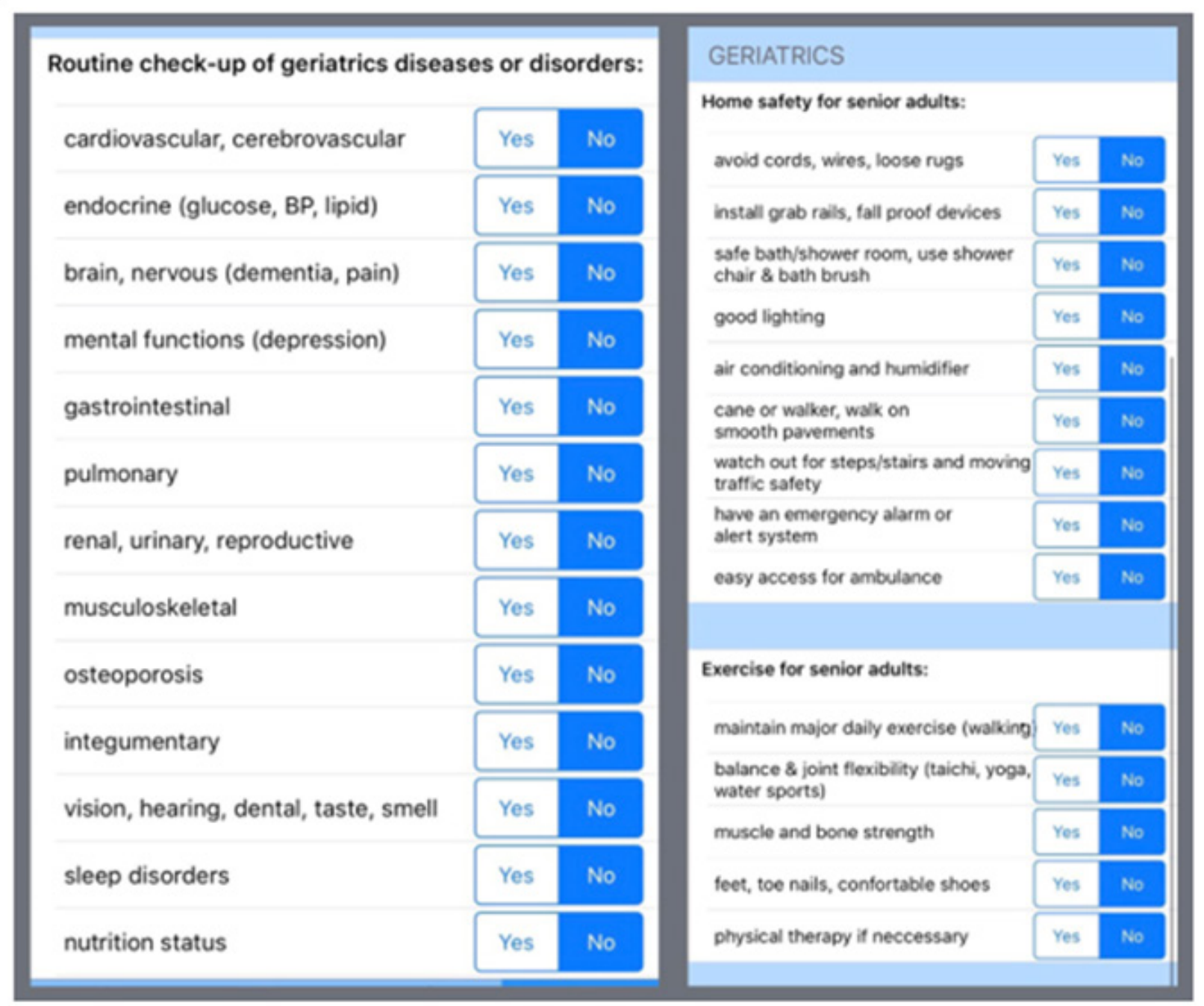

Figure 5 Three additional components for Geriatrics concerns.

\section{Conclusion}

Geriatrics is a summarized or combined branch of nearly all of the internal medical branches. Metabolic disorder is the fundamental source of many chronic diseases. High glucose level is also the root cause of diabetes which leads into many complications of the internal organs.

Although these six metabolic lifestyle categories, which include food \& diet, exercise, sleep, stress, water intake, routine life pattern, and home safety are critical to endocrine diseases, they also serve as major risk factors for longevity. As shown in Figure 6 (from Bradley Wilcox, MD, JABSOM University of Hawaii), even at age 90, there are major differences between survival probability of $77 \%$ with no risk factors and survival probability of $43 \%$ with $5+$ risk factors. Therefore, in order to have longevity, it is never too late to maintain a good lifestyle by paying attention to these metabolic lifestyle categories.

By using the GH-Method: math-physical medicine to expand his research results for chronic diseases into the area of geriatrics is a natural extension of his medical research work. His previous mathematical and physical models, quantitative analytics and engineering techniques, various statistical analysis tools are all extremely useful for his study and research of geriatrics. 


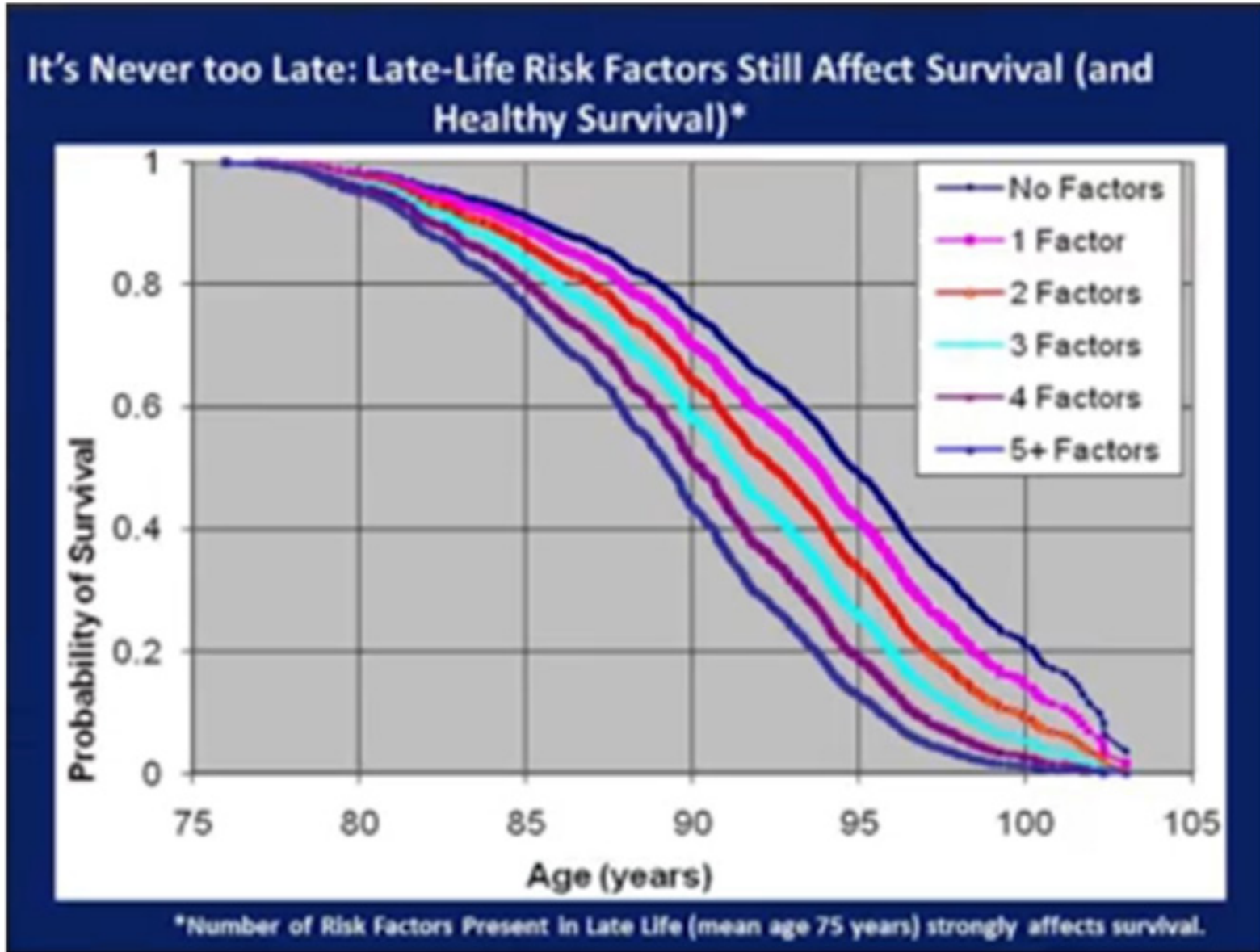

Figure 6 Longevity life risk factors affect probability of survival.

\section{Acknowledgments}

First and foremost, the author wishes to express his sincere appreciation to a very important person in his life, Professor Norman Jones at MIT and University of Liverpool. Not only did he give him the opportunity to study for his PhD at MIT, but he also trained him extensively on how to solve difficult problems and conduct any basic scientific research with a big vision, pure heart, and integrity.

The author would also like to thank Professor James Andrews at the
University of Iowa. He helped and supported him tremendously when he first came to the United States. He believed in him and prepared him to build his solid engineering and computer science foundation. $\mathrm{He}$ is forever grateful to his mentor, who has a kind heart and guided him during his undergraduate and master's degree work at Iowa.

\section{Conflicts of interest}

The authors declare have no conflict of interest about the publication of this paper. 